\title{
Ionic-covalent transition in titanium oxides
}

\author{
Carmen Sousa and Francesc Illas \\ Departament de Química Física, Grup de Química Quàntica, Universitat de Barcelona, C/Martí i Franquès 1, 08028 Barcelona, Spain
}

(Received 6 May 1994)

\begin{abstract}
The nature of the chemical bond in three titanium oxides of different crystal structure and different formal oxidation state has been studied by means of the $a b$ initio cluster-model approach. The covalent and ionic contributions to the bond have been measured from different theoretical techniques. All the analysis is consistent with an increasing of covalence in the $\mathrm{TiO}, \mathrm{Ti}_{2} \mathrm{O}_{3}$, and $\mathrm{TiO}_{2}$ series as expected from chemical intuition. Moreover, the use of the $a b$ initio cluster-model approach combined with different theoretical techniques has permitted us to quantify the degree of ionic character, showing that while TiO can approximately be described as an ionic compound, $\mathrm{TiO}_{2}$ is better viewed as a rather covalent oxide.
\end{abstract}

\section{INTRODUCTION}

The study of the electronic structure and related properties of transition-metal oxides is one of the most fascinating and outstanding problems in solid-state physics. These materials have attracted great interest because of their electrical properties, which present almost all the different possibilities: insulators such as $\mathrm{NiO}$, semiconductors such as $\mathrm{Ti}_{2} \mathrm{O}_{3}$, and superconductors such as $\mathrm{SrTiO}_{3}{ }^{1}$ The importance of transition-metal oxides in technological applications has motivated several experimental and theoretical studies. However, few $a b$ initio studies have been done on the electronic structure of these compounds, mainly due to the complex structures containing many atoms per unit cell.

One of the common difficulties in the study of transition-metal oxides is that the different chemical and electrical behavior may depend on the transition metal, the oxidation state, and the crystal structure. Titanium oxides are particularly well suited for a theoretical systematic study because they share the same transition metal but present different structures and properties. In fact, three different titanium oxides, with the titanium atom having different oxidation states of $+2,+3$, and +4 , have been found to show different crystal structures and electrical behavior. Thus, titanium (II) oxide, TiO, is metallic, crystallizes in the rocksalt structure, and both $\mathrm{O}$ and $\mathrm{Ti}$ ions are octahedrally coordinated. Titanium (III) oxide, $\mathrm{Ti}_{2} \mathrm{O}_{3}$, is a semiconductor, exhibits the corundum structure, and presents a temperature-dependent semiconductor-to-metal transition being metallic above $400^{\circ} \mathrm{C} .^{2}$ Finally, titanium (IV) oxide, $\mathrm{TiO}_{2}$, in the most stable modification, the rutile structure, exhibits typical insulator properties.

Rutile has been the subject of great interest precisely because it is an insulator with a wide band gap, about $3.0-3.3 \mathrm{eV},{ }^{3}$ but becomes a semiconductor when there is an oxygen deficiency. ${ }^{4}$ The electronic structure of rutile has been widely studied by different experimental spectroscopic techniques, including ultraviolet photoelectron spectroscopy (UPS), ${ }^{5,6} \mathrm{x}$-ray photoelectron spectroscopy (XPS), ${ }^{7-9}$ and electron-energy-loss spectroscopy
(EELS). ${ }^{10,11}$ From the theoretical point of view, several studies have been performed to obtain the optimized lattice parameters ${ }^{12,13}$ and also the cohesive energy of the crystal. ${ }^{14,15}$ A detailed study of the electronic structure of rutile has been carried out by Silvi and coworkers $^{16-17}$ using a pseudopotential periodic HartreeFock approach. This calculation predicts an optimized geometry in good agreement with experiment. The analysis of electron-density maps and population analysis show an involvement of the titanium $d$ electrons in the bonding. The Mulliken population analysis shows a partially ionic character in $\mathrm{TiO}_{2}$, with net atomic charges of +2.6 and -1.3 for $\mathrm{Ti}$ and $\mathrm{O}$, respectively. Compared to the formal point charge of +4 this result suggests $65 \%$ ionic character. Bader analysis of charge density has also been applied to rutile; the result obtained shows an ionicity of $75 \%,{ }^{17}$ similar to that derived from the Mulliken population analysis. The study of the electronic band structure indicates that the conduction band is predominantly of titanium $3 d$ character, containing significant contributions of $2 s$ and $2 p$ oxygen orbitals. A significant $\operatorname{Ti}(3 d)-\mathrm{O}(2 p)$ hybridization was also predicted from a cluster-based local-density approach which explicitly included the interaction of the cluster with the crystal environment. ${ }^{18}$ Finally, let us point out that the same qualitative measure of the ionicity appears from Pauling's electronegativity scale. Using this empirical approach Glassford and Chelikowsky ${ }^{15}$ estimate $60 \%$ ionic character in the Ti-O bond.

The exhaustive description of the properties of rutile in the literature contrasts with the lack of data for other titanium oxides. In this work, we present an $a b$ initio cluster-model study of different titanium oxides, TiO, $\mathrm{Ti}_{2} \mathrm{O}_{3}$, and $\mathrm{TiO}_{2}$, where titanium presents different formal charges which range from 2 to 4 . This study allows us to analyze the Ti-O chemical bonding in these compounds and to determine the different degree of ionicity and covalency in each oxide. The analyses performed include a decomposition of the interaction energy between cations and anions corresponding to each crystalline structure, the analysis of the core-level binding-energy shifts, and the analysis of different $a b$ initio wave functions of in- 
creasing accuracy. We must point out that none of these analyses can give definitive information about the character of a bond, but all being consistent can give a clear indication of the nature of the chemical bonding.

As expected, the results obtained are consistent with an increase of covalence as the oxidation number of $\mathrm{Ti}$ increases, i.e., along the series $\mathrm{TiO}, \mathrm{Ti}_{2} \mathrm{O}_{3}, \mathrm{TiO}_{2}$. The present study permits one to quantify this trend along the series, to determine the different contributions to the bond, and to show unequivocally that $\mathrm{TiO}$ and $\mathrm{Ti}_{2} \mathrm{O}_{3}$ are essentially ionic compounds, whereas a clear buildup of a covalent chemical bond exists in rutile.

\section{CLUSTER MODELS}

A cluster-model approach has been chosen to carry out a systematic study of the chemical bond in titanium oxides and to investigate a possible ionic-to-covalent transition as the oxidation state of the metal cation grows from +2 to +4 . The use of a finite model permits us to study local properties and to use state-of-the-art theoretical approaches. The nature of the chemical bonding between the two kinds of atoms (or ions) forming an "ionic" crystal such as a transition-metal oxide is precisely the local property that we want to analyze.

The clusters chosen to represent the three bulk oxides contain one titanium atom and the six nearest-neighbor oxygen atoms placed at the corresponding crystal positions with Ti-O distances equal to the experimental values $^{19}$ for each crystalline structure. The cluster models considered are presented in Fig. 1. The number of electrons explicitly involved in the calculations is that corresponding to an ionic configuration. Thus, we consider 10 electrons for each $\mathrm{O}^{2-}$ anion and 20,19 , and 18 electrons for the $\mathrm{Ti}^{2+}, \mathrm{Ti}^{3+}$, and $\mathrm{Ti}^{4+}$ titanium cations in TiO, $\mathrm{Ti}_{2} \mathrm{O}_{3}$, and $\mathrm{TiO}_{2}$, respectively. We must point out that this choice does not represent any constraint to our study. The $a b$ initio wave functions that we will use (vide infra) are flexible enough to allow any redistribution of the starting electron density. Thus, if the cluster total energy is variationally lower for a distribution where oxygen atoms prefer a -1 net charge, a charge flow from the oxygens to the metal atoms will occur and will be reflected in the final electronic wave function.

For $\mathrm{TiO}$, the cluster model considered is $\left[\mathrm{TiO}_{6}\right]^{10-}$ plus a grid of 343 optimized point charges (OPC's) placed at the lattice positions, to reproduce the Madelung potential in the cluster region. ${ }^{20}$ For the titanium (III) oxide, $\mathrm{Ti}_{2} \mathrm{O}_{3}$, the cluster model is $\left[\mathrm{TiO}_{6}\right]^{9-}$ with two inequivalent Ti-O bond lengths of 2.01 and $2.08 \AA$. This cluster has been embedded in an array of 248 OPC's to represent the Madelung potential corresponding to a corundum crystalline structure. Finally, titanium (IV) oxide, in the rutile structure, has been represented by a $\left[\mathrm{TiO}_{6}\right]^{8-}$ cluster model where the central $\mathrm{Ti}^{4+}$ is surrounded by two apical and four equatorial $\mathrm{O}^{2-}$ anions giving two Ti-O bond distances of 1.969 and $1.884 \AA$, respectively. ${ }^{19}$ The crystal environment has been described including, again, an array of 477 OPC's to represent the Madelung potential corresponding to a full ionic situation in rutile.

\section{WAVE FUNCTION AND BASIS SETS}

For each one of the cluster models described in the previous section we have obtained $a b$ initio all-electron Hartree-Fock cluster wave functions following the wellknown linear combination of atomic orbitals molecular orbital (LCAO-MO) self-consistent field (SCF) procedure where the molecular orbitals are expanded in terms of a set of basis functions. Here, the MO's have been expanded in terms of contracted Gaussian-type orbitals (CGTO's). The basis set for the $\mathrm{O}^{2-}$ ions is $(11 s 7 p / 5 s 3 p)$ and has been taken from Pacchioni, Cogliandro, and Bagus. ${ }^{21}$ For the titanium atoms the basis set is basically the one reported by Huzinaga, ${ }^{22}$ augmented with a representation of the outer $4 s$ and $3 d$ orbitals; the final basis set may be represented at $(14 s 8 p 5 d / 8 s 4 p 2 d)$.

(a)

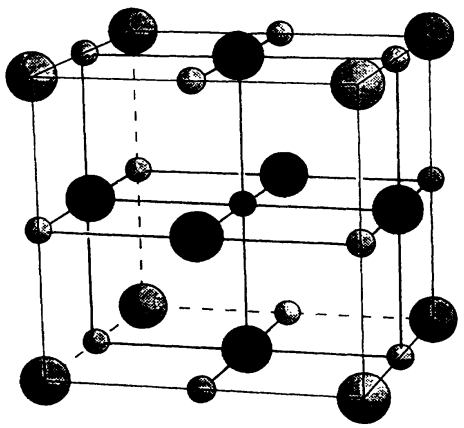

(b)

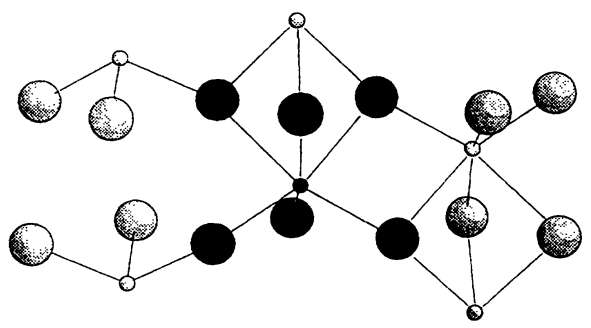

(c)

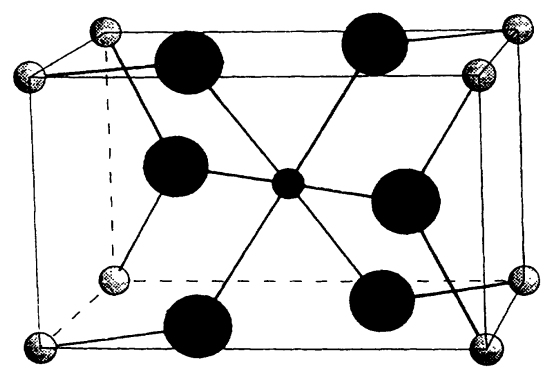

FIG. 1. Representation of the cluster models used to represent (a) $\mathrm{TiO}$, (b) $\mathrm{Ti}_{2} \mathrm{O}_{3}$, and (c) $\mathrm{TiO}_{2}$. Filled circles correspond to the ions in the cluster model and empty circles to some of the point charges used to provide an appropriate environment. Small and large circles represent the $\mathrm{Ti}$ cations and $\mathrm{O}^{2-}$ anions, respectively. 
As stated previously, an initial $1 s^{2} 2 s^{2} 2 p^{6}$ electronic configuration is assumed for the $\mathrm{O}^{2-}$ ions whereas [Ar] $3 d^{2}$, [Ar] $3 d^{1}$, and [Ar] $3 d^{0}$ starting electronic configurations are assumed for $\mathrm{Ti}^{2+}, \mathrm{Ti}^{3+}$, and $\mathrm{Ti}^{4+}$, respectively.

In the case of rutile we have also obtained more sophisticated electronic wave functions which explicitly include the electronic correlation effects. In this case the MO's used to construct the correlated multireference configuration-interaction (MRCI) wave functions were determined considering nonempirical pseudopotentials ${ }^{23}$ for the $\mathrm{O}$ atoms. In this case a $(6 s 6 p) /[2 s 2 p]$ basis set was used to describe the oxygen atoms. The MRCI wave functions were obtained following the CIPSI algorithm. ${ }^{24-28}$

All calculations were carried out using a locally modified version of the HONDO-CIPSI chain of programs. ${ }^{29}$

\section{ENERGY DECOMPOSITION}

In this section we will determine the different contributions to the final SCF energy corresponding to a given cluster model. To this end we start from a purely ionic configuration and use the constrained space orbital variation (CSOV) method mo-32 $^{30}$ to determine the cluster SCF wave function with various constraints. This procedure permits us to identify the relative magnitudes of the electrostatic, covalent, and polarization contributions which lead from a purely ionic starting point to the final SCF wave function. To obtain the starting ionic wave function, hereafter referred to as the frozen orbital (FO), we have previously determined the SCF wave functions for two well-defined interacting fragments in each cluster model simulating the different titanium oxides.

Thus, for the $\left[\mathrm{TiO}_{6}\right]^{10-}$ cluster model used to represent $\mathrm{TiO}$ we have chosen the following units: the $\mathrm{Ti}^{2+}$ embedded in the field created by the corresponding OPC's, referred to as $\mathrm{NaCl}$ OPC's, and the $\left[\mathrm{O}_{6}\right]^{12-}$ unit compared with an effective core potential ${ }^{23}$ (ECP) to represent the central titanium cation, plus the same array of $\mathrm{NaCl}$ OPC's. The consideration of this ECP accounts for effects due to the finite size of the cation, as has been discussed elsewhere. ${ }^{33,34}$ For $\mathrm{Ti}_{2} \mathrm{O}_{3}$, represented by the cluster $\left[\mathrm{TiO}_{6}\right]^{9-}$, the considered fragments were $\mathrm{Ti}^{3+}$ plus the OPC's corresponding to the corundum structure, and the $\left[\mathrm{O}_{6}\right]^{12-}$ unit with a central Ti ECP and embedded in corundum OPC's. Similarly, $\mathrm{Ti}^{4+}$ plus rutile OPC's and $\left[\mathrm{O}_{6}\right]^{12-}$ with a Ti ECP plus rutile OPC's were considered to give the $\left[\mathrm{TiO}_{6}\right]^{8-}$ cluster model to represent $\mathrm{TiO}_{2}$.

The starting point of the CSOV procedure, or the frozen-orbital step, is determined by superimposing the two interacting fragments at the cluster nuclear positions. The initial FO wave function contains the attractive terms arising from the Madelung potential plus the repulsive ones arising from the Pauli repulsion between the electronic densities of the two units which have not been allowed to vary (except for the orthogonalization between the MO's of the two units required to have a FO Slater determinant made of orthonormalized MO's). It is clear that this FO wave function corresponds to a purely ionic description. Because our goal is to determine the degree of ionicity in each compound, the total energy at this step appears to be the natural zero reference energy. In the following steps, the units are allowed to vary. The first variation allows the different titanium cations to polarize within the space of the occupied and virtual $\mathrm{Ti}$ orbitals, denoted as $V(\mathrm{Ti} ; \mathrm{Ti})$ in Table I. As expected from the small polarizability of the cations, the energy contribution associated with this step is not significant. The next CSOV step measures the extent of charge transfer from the occupied $\mathrm{Ti}$ orbitals to the $\left[\mathrm{O}_{6}\right]^{12-}$ virtual orbitals; $V(\mathrm{Ti}$;all $)$ in Table I. At this point the $\left[\mathrm{O}_{6}\right]^{12-}$ unit has not varied; the valence shell of each oxygen is completely filled, and therefore this energy contribution can only be due to a basis-set superposition error (BSSE), being more important in the case of $\mathrm{TiO}$ and $\mathrm{Ti}_{2} \mathrm{O}_{3}$, where the $3 d$ orbitals are partially occupied. In the following variation, $V(\mathrm{O} ; \mathrm{O})$, the $\mathrm{O}^{2-}$ anions are allowed to polarize. The energy decrease associated with $V(O ; O)$ mainly contributes to the reduction of the initial Pauli repulsion; it is about 1 $\mathrm{eV}$ for $\mathrm{TiO}, 0.7 \mathrm{eV}$ for $\mathrm{Ti}_{2} \mathrm{O}_{3}$, and approximately $0.6 \mathrm{eV}$ for $\mathrm{TiO}_{2}$. Part of the fairly different energy stabilization associated with the oxygen polarization can be due to the fact that the starting $\left[\mathrm{O}_{6}\right]^{12-}$ electron density has been obtained using a model having a central Ti ECP, which, in fact, corresponds to an [Ar] configuration. The spatial extent of this ECP is, therefore, more similar to that of the $\mathrm{Ti}^{4+}$ cation than to that of $\mathrm{Ti}^{2+}$; thus the interaction of the oxygen orbitals with the real cation orbitals is larger in $\mathrm{TiO}$ than it is in $\mathrm{TiO}_{2}$, with $\mathrm{Ti}_{2} \mathrm{O}_{3}$ presenting an intermediate value.

In the next step of the CSOV, $V(O ;$ all $)$, the covalent interaction is taken into account. The occupied $\left[\mathrm{O}_{6}\right]^{12-}$ orbitals are allowed to mix with the empty cation orbitals, and thus charge transfer from $\left[\mathrm{O}_{6}\right]^{12-}$ to the titanium cations is permitted. The energy contribution due to this covalent mixing is the most important contribution, about $1.5 \mathrm{eV}$ for $\mathrm{TiO}, 4 \mathrm{eV}$ for $\mathrm{Ti}_{2} \mathrm{O}_{3}$, and larger than 10 eV for $\mathrm{TiO}_{2}$. These large contributions indicate an important degree of covalence for all these titanium oxides, this being significantly larger for rutile. However, the computed $V(\mathrm{O}$; all $)$ contributions can be affected, to some extent, by a BSSE due to the oxygen basis-set limitation. Nevertheless, it is very unlikely that this BSSE could affect significantly the covalent contribution and particularly the trend of the covalence along these three titanium oxides. In the case of $\mathrm{TiO}$ and $\mathrm{Ti}_{2} \mathrm{O}_{3}$ there is an additional contribution. The $\mathrm{Ti}$ occupied open-shell orbitals

TABLE I. Energy gain (in eV) at each CSOV step for the models considered to represent $\mathrm{TiO}, \mathrm{Ti}_{2} \mathrm{O}_{3}$, and $\mathrm{TiO}_{2}$ (see text).

\begin{tabular}{lccr}
\hline \multicolumn{1}{c}{ CSOV step } & $\mathrm{TiO}$ & $\mathrm{Ti}_{2} \mathrm{O}_{3}$ & $\mathrm{TiO}_{2}$ \\
\hline$V(\mathrm{Ti} ; \mathrm{Ti})$ & 0.036 & 0.026 & 0.012 \\
$V(\mathrm{Ti} ;$ all $)$ & 0.096 & 0.054 & 0.037 \\
$V(\mathrm{O} ; \mathrm{O})$ & 1.082 & 0.705 & 0.651 \\
$V(\mathrm{O} ;$ all $)$ & 1.459 & 3.981 & 10.447 \\
$V($ op;cl) & 0.052 & 0.109 & 0.000 \\
Additional terms & 0.149 & 0.137 & 0.315 \\
\hline
\end{tabular}


can mix with the $\left[\mathrm{O}_{6}\right]^{12-}$ occupied closed-shell orbitals giving rise to small energy contributions, denoted as $V(o p ; c l)$ in Table I. The sum of all these contributions is close to the total SCF interaction energy obtained with an unconstrained calculation, except for $\mathrm{TiO}_{2}$. In this case, there is an additional stabilization of about $0.3 \mathrm{eV}$ in going from the $V(O$;all) to the final SCF step (see Table I). This indicates that some coupling occurs among these bonding mechanisms. This is because the order of the variations is, in principle, arbitrary. ${ }^{32}$ Therefore, the CSOV analysis for rutile was carried out again allowing the oxygens to vary first. The results show that all the contributions remained practically unchanged except the $\mathrm{Ti}^{4+}$ polarization contribution which becomes larger by about $0.4 \mathrm{eV}$. This stabilization contribution is due to the $\mathrm{Ti}^{4+}$ polarization which now occurs after the important charge donation from the $\left[\mathrm{O}_{6}\right]^{12-}$ occupied orbitals to the $\mathrm{Ti}^{4+}$ unoccupied orbitals, involving $10 \mathrm{eV}$ of energy stabilization. In this second CSOV decomposition, the sum of the bonding contributions is nearly equal to the interaction energy obtained with an unconstrained SCF calculation. This proves that the previous energy decomposition is correct.

The main conclusion from these CSOV analyses is that the most important energy contribution is due to the charge donation of $\left[\mathrm{O}_{6}\right]^{12-}$ to the titanium cations. This contribution is important in all the oxides and increases as the oxidation number or formal charge on the $\mathrm{Ti}$ increases. These results suggest an important degree of covalent Ti-O bonding in these oxides. Similar studies on alkaline-earth oxides ${ }^{33}$ and on aluminum oxide ${ }^{34}$ show stabilization energies by covalent interaction of about 0.9 $\mathrm{eV}$ for $\mathrm{BaO}$, the most covalent oxide among the alkalineearth series, and of about $1.6 \mathrm{eV}$ for $\mathrm{Al}_{2} \mathrm{O}_{3}$. The degree of covalence in these oxides has also been reported in Refs. 33 and 34, where it has been shown that the alkalineearth oxides are practically fully ionic compounds, $\mathrm{BaO}$ being the least ionic of the series, whereas $\mathrm{Al}_{2} \mathrm{O}_{3}$ presents not less than $90 \%$ ionic character. In these compounds, the real charge on the cation is close to the formal one. However, in the three titanium oxides under study it is very unlikely that the real charge on titanium would be close to the formal oxidation state, especially in the case of $\mathrm{TiO}_{2}$ where $\mathrm{Ti}$ assumes an oxidation state of 4 .

Finally, we would like to point out that the CSOV analysis provides information about the different energy contributions to the final SCF energy. To obtain a relationship between the energy stabilization contribution and the real degree of covalent mixing in the titanium oxides further analyses are needed; these are presented in the following sections.

\section{CORE-LEVEL BINDING-ENERGY SHIFTS}

A common measure of the importance of covalent effects in narrow-band systems is the analysis of experimental $x$-ray photoelectron spectra. The main idea is that charge transfer from the anions to the metal cation will lead to important shifts in the core-level binding energies (BE's) of the metal when compared with either the bulk metal or the gas phase BE's. In fact, when electron- ic charge is transferred from the anion occupied orbitals to the cation empty orbitals, a decrease in the core-level BE's of the cation is observed, because the increased electron density screens the core levels of the metal. ${ }^{35,36}$ However, the relationship between charge transfer and experimental BE shifts is not straightforward, because many physical mechanisms may be involved. ${ }^{37,38}$

From the theoretical point of view, and in the framework of the cluster-model approach, it is possible to evaluate the initial-state metal core-level BE shifts by simply taking the energy of the orbital considered, following Koopman's theorem. Then we can make use of the CSOV technique to separate the different contribution to the BE shifts. In particular, the variation of the core level BE's at the $V(O$;all $)$ step can be related to the extent of covalent mixing.

We have computed the shifts between the $V(O$;all $)$ step and the previous $V(O ; O)$ step for the $2 p$ core levels of the $\mathrm{Ti}$ cation in the different oxides. The shifts are always negative, indicating an increase of the electron density of the metal at the $V(O ;$;all $)$ step that leads to a smaller $\mathrm{BE}$ after the donation step. The shift is of about $-1.8 \mathrm{eV}$ for TiO, $-5.0 \mathrm{eV}$ for $\mathrm{Ti}_{2} \mathrm{O}_{3}$, and approximately $-11 \mathrm{eV}$ for $\mathrm{TiO}_{2}$, in accord with an increase in the covalent character from $\mathrm{TiO}$ to $\mathrm{TiO}_{2}$. These data are in agreement with the increasing importance of the stabilization by charge donation from the anions to the cations. Again, a specific measure of covalence cannot be obtained from the analysis of BE shifts. Covalent effects on the core-level shifts are almost ten times more important for $\mathrm{TiO}_{2}$ than for TiO; still this fact does not permit one to quantify the amount of covalence in each compound.

\section{ANALYSIS OF THE ELECTRONIC WAVE FUNCTION}

The CSOV procedure allows us to obtain information on the energy associated with the different contributions to a given chemical process. However, we are interested not only in the energy gain at each CSOV step, but also in its effect on the electronic structure. Our purpose here is to relate the energy stabilization observed at the donation $V(O$;all $)$ step, and the actual degree of charge transfer between the two interacting fragments. The same argument holds for the core-level BE shifts. The goal is not only to predict the proper trend as in the experimental XPS data but also to relate a BE shift with changes in the electronic structure.

To analyze the SCF wave function we will once more make use of the CSOV decomposition. The main idea here is to obtain the wave function at each CSOV step and use the fact that the wave function obtained at the first CSOV step, the FO step, corresponds to a fully ionic description. Thus we can see how the wave function changes by computing the overlap between the FO wave function and each one of the wave functions corresponding to each different step, $\left\langle\psi_{\mathrm{FO}} \mid \psi_{i}\right\rangle$. This overlap is an unambiguous measure of the similarity of the two wave functions and gives a quantitative measure of the ionic character of the wave function associated with each CSOV step. In fact, if the overlap between the SCF wave 
function and the FO ionic one is close to 1 we have a clear indication that the two wave functions are very similar. The square of $\left\langle\psi_{\mathrm{FO}} \mid \psi_{i}\right\rangle$ multiplied by 100 may be viewed as the percent of ionic character contained in the final SCF wave function.

In Table II we present, for the three Ti oxides, the results for the $\left\langle\psi_{\mathrm{FO}} \mid \psi_{i}\right\rangle$ integral at each step of the CSOV decomposition. We are particularly interested in the overlap between the FO wave function, i.e., the fully ionic description, and the wave function obtained after the charge transfer is allowed. The results contained in Table II show that the most important variation of the $\left\langle\psi_{\mathrm{FO}} \mid \psi_{i}\right\rangle$ overlap is obtained precisely at the $V(\mathrm{O}$;all) step, indicating that there is an important charge donation from the oxygen occupied orbitals to the cation unoccupied orbitals. The unconstrained SCF wave function contains about $85 \%$ of ionic description for $\mathrm{TiO}$, $78 \%$ for $\mathrm{Ti}_{2} \mathrm{O}_{3}$, and approximately $57 \%$ for $\mathrm{TiO}_{2}$. It is important to notice that these values correspond to a lower bound of the ionicity in these compounds since we have included all the physical effects which contribute to decrease the overlap integral, but which are by no means due to an increase of the covalent character of the chemical bond. If we take the percent of the purely ionic configuration as a measure of the net charge of titanium, we will obtain a value of +1.7 for $\mathrm{TiO},+2.4$ for $\mathrm{Ti}_{2} \mathrm{O}_{3}$, and +2.3 for $\mathrm{TiO}_{2}$. The result obtained for $\mathrm{TiO}_{2}$ is comparable with other results reported in the literature. ${ }^{15,16}$ Thus, Silvi et $a l .{ }^{16}$ have estimated a net charge of +2.6 for the titanium atom and Glassford and Chelikowsky ${ }^{15}$ have reported $40 \%$ covalent character of the Ti-O bonding in $\mathrm{TiO}_{2}$, close to our measure of $57 \%$ ionic character. The results obtained from this analysis indicate an increase of the covalent character in going from $\mathrm{TiO}$ to $\mathrm{Ti}_{2} \mathrm{O}_{3}$ to $\mathrm{TiO}_{2}$.

We can compare these results with previous studies on alkaline-earth oxides and corundum, ${ }^{34-36,39,40}$ which have been proved to be highly ionic oxides. Thus, TiO turns out to be less ionic than the alkaline-earth oxides, which present the same crystal structure. For the alkaline-earth oxides the amount of ionic character is always greater than $95 \%$, whereas for $\mathrm{TiO}$ it does not exceed $85 \%$. Similarly, $\mathrm{Ti}_{2} \mathrm{O}_{3}$ is about $10-15 \%$ more covalent than $\mathrm{Al}_{2} \mathrm{O}_{3}$, both having the corundum structure.

\section{ELECTRONIC CORRELATION EFFECTS}

The analyses in the preceding sections have shown a significant covalent character of the $\mathrm{Ti}-\mathrm{O}$ bond in the titanium oxides, especially in the case of $\mathrm{TiO}_{2}$ which turns out to be about $43 \%$ covalent. This result may also be interpreted in terms of the more intuitive valence-bond (VB) theory. In the VB approach the electronic structure of a given compound is usually written in terms of a configuration interaction between different Slater determinants, written in an atomic basis set, each having a well-defined physical meaning (see Refs. 39 and 41). The chemical bond appears then as resonance between different VB structures, where each of these structures has a weight or contribution to the final wave function. The results in the previous section can be related to the VB theory because the contribution of the FO wave function to the SCF one is just the contribution of the ionic forms. It may be properly argued that the analyses in the previous sections have been done using uncorrelated, SCF, wave functions and that the weight of the ionic contributions could be different for a more accurate wave function.

In order to study the effects of electronic correlation on our measure of the ionicity we have determined correlated multireference configuration-interaction wave functions for the more covalent of the titanium oxides, $\mathrm{TiO}_{2}$. To include the most important determinants in our variational MRCI wave function, we have used a selection criterion based on the CIPSI algorithm. ${ }^{24-28}$ For interpretative purposes our MRCI expansion is constructed from the ionic, $\left|\psi_{\mathrm{FO}}\right\rangle$, FO determinant. The reference space was iteratively constructed leading to a zeroth-order wave function containing the more important 205 single and double excitations out of $\left|\psi_{\mathrm{FO}}\right\rangle$. The MRCI wave function was constructed from this reference by generation of all single and double excitations from each reference determinant. Thus the MRCI wave function may be written as

$$
\begin{aligned}
\left|\psi_{\mathrm{MRCI}}\right\rangle= & c_{\mathrm{FO}}\left|\psi_{\mathrm{FO}}\right\rangle+\sum_{i \in \mathrm{FO}} \sum_{j \notin \mathrm{FO}} c_{i j} a_{j}^{\dagger} a_{i}\left|\psi_{\mathrm{FO}}\right\rangle \\
& +\sum_{i, j \in \mathrm{FO}} \sum_{k, l \notin \mathrm{FO}} c_{i j} c_{k l} a_{k}^{\dagger} a_{l}^{\dagger} a_{j} a_{i}\left|\psi_{\mathrm{FO}}\right\rangle+\cdots
\end{aligned}
$$

TABLE II. The overlap integral between the FO wave function and the corresponding wave function at each CSOV step, $\left\langle\psi_{\mathrm{FO}} \mid \psi_{i}\right\rangle$. The percent importance of the FO wave function at the different steps is also given.

\begin{tabular}{lcccccc}
\hline \multicolumn{1}{c}{ CSOV step } & \multicolumn{2}{c}{$\mathrm{TiO}$} & \multicolumn{2}{c}{$\mathrm{Ti}_{2} \mathrm{O}_{3}$} & \multicolumn{2}{c}{$\mathrm{TiO}_{2}$} \\
& $\left\langle\psi_{\mathrm{FO}} \mid \psi_{i}\right\rangle^{2}$ & $\%$ & $\left\langle\psi_{\mathrm{FO}} \mid \psi_{i}\right\rangle$ & $\%$ & $\left\langle\psi_{\mathrm{FO}} \mid \psi_{i}\right\rangle$ & $\%$ \\
\hline Frozen orbital & 1.0000 & 100 & 1.0000 & 100 & 1.0000 & 100 \\
$V(\mathrm{Ti} ; \mathrm{Ti})$ & 0.9998 & 99.99 & 0.9997 & 99.95 & 0.9999 & 99.99 \\
$V(\mathrm{Ti} ;$ all $)$ & 0.9973 & 99.46 & 0.9991 & 99.81 & 0.9997 & 99.94 \\
$V(\mathrm{O} ; \mathrm{O})$ & 0.9685 & 93.80 & 0.9838 & 96.78 & 0.9896 & 97.94 \\
$V(\mathrm{O} ;$;all $)$ & 0.9373 & 87.85 & 0.8961 & 80.31 & 0.7758 & 60.19 \\
$V($ op;cl) & 0.9362 & 87.65 & 0.8934 & 79.81 & 0.7758 & 60.19 \\
Additional terms & 0.9242 & 85.41 & 0.8845 & 78.23 & 0.7569 & 57.29 \\
\hline \hline
\end{tabular}


and includes up to quadrupole excitations. The MRCI expansions thus generated are usually too large to be solved variationally. Therefore we use a threshold criterion to truncate the total MRCI expansion. We have considered two different expansions including only the determinants with larger first-order contributions to the MRCI wave function (vide infra).

In Eq. (1) $c_{\mathrm{FO}}$ is the coefficient of the ionic determinant to the final MRCI expansion. For a fully ionic description, one would expect $c_{\mathrm{FO}}=1$, and any deviation from this value would seem to indicate a deviation with respect to the ionic model. However, the analysis of the variation of $c_{\mathrm{FO}}$ is not straightforward and several effects have to be taken into account. For instance, if for the SCF wave function $\left|\psi_{\mathrm{SCF}}\right\rangle$ we use a development similar to that indicated in Eq. (1) we will have (up to first order)

$$
\left|\psi_{\mathrm{SCF}}\right\rangle=c_{\mathrm{FO}}\left|\psi_{\mathrm{FO}}\right\rangle+\sum_{i \in \mathrm{FO}} \sum_{j \notin \mathrm{FO}} c_{i j} a_{j}^{\dagger} a_{i}\left|\psi_{\mathrm{FO}}\right\rangle
$$

and one can be tempted to say that $c_{\mathrm{FO}}$ in Eq. (2) gives an estimate of the contribution of the ionic description of the SCF wave function. In fact, the value of $c_{\mathrm{FO}}$ is now identical (again up to first order) to the $\left\langle\psi_{\mathrm{FO}} \mid \psi_{\mathrm{SCF}}\right\rangle$ overlap integral discussed in the previous section. We must point out that because monoexcitations in Eq. (2) can involve orbitals in the same unit or not, a decrease in $c_{\mathrm{FO}}$ may be due to intraunit polarization or to interunit charge-transfer donation as obtained when the CSOV procedure is used (see results in Table II). However, the numerical results in Table II show that the only important change occurs when donation to the cation is allowed; changes in the wave function due to the intraunit polarization effects are less than $3 \%$. Therefore $\left\langle\psi_{\mathrm{FO}} \mid \psi_{\mathrm{SCF}}\right\rangle$, or equivalently $c_{\mathrm{FO}}$, can be effectively taken as a measure of the ionic contribution to the SCF wave function. Now, if we wish to understand the effect of electronic correlation we have to compare $c_{\mathrm{FO}}$ in Eq. (2) with the value computed using Eq. (1). Still one must realize that not all the excitations considered in Eq. (1) contribute to the electronic correlation effects because $\left|\psi_{\mathrm{FO}}\right\rangle$ is not the SCF wave function and the single excitations of $\left|\psi_{\mathrm{FO}}\right\rangle$ only serve to recover the SCF determinant (see Ref. 34). Therefore we can take the difference in $c_{\text {FO }}$ obtained from Eqs. (1) and (2) as a measure of the importance of the change in the electronic wave function due to correlation effects.

The first MRCI expansion we consider contains 13424 determinants and the projection of $\left|\psi_{\mathrm{FO}}\right\rangle$ in the $\left|\psi_{\text {MRCI }}\right\rangle$ wave function is 0.748 or $56.0 \%$. This result is very similar to that obtained in the analysis of the SCF wave function reported above, which was $57.3 \%$. This result indicates that the inclusion of the correlation effects does not significantly change the nature of the bonding. The second MRCI expansion involves 69608 determinants, five times larger than the previous one. The contribution of $\left|\psi_{\mathrm{FO}}\right\rangle$ to the $\left|\psi_{\mathrm{MRCI}}\right\rangle$ is 0.733 or, equivalently, $53.7 \%$. Therefore a rather large increase of the number of determinants included in $\left|\psi_{\text {MRCI }}\right\rangle$ produces only about $4 \%$ decrease in the contribution of the purely ionic configuration. Moreover, it should be noted that there are many configurations which correspond to intraunit dynamical electron correlation, i.e., excitations from the oxygen $2 p$ orbitals to the oxygen virtual space, that lead to a decrease of $c_{\mathrm{FO}}$ which is by no means due to an increase of the covalent character of the bond.

The above results clearly show that inclusion of the electron correlation effects does not significantly affect the degree of covalence and leads to the same conclusion obtained when a SCF description is considered, as has previously been reported for the alkaline-earth oxides ${ }^{40}$ and, also, for aluminium oxide. ${ }^{34}$

\section{CONCLUSIONS}

The nature of the chemical bond in three titanium oxides of different crystal structure and different formal oxidation state has been studied by means of the $a b$ initio cluster-model approach. The covalent and ionic contributions to the bond have been measured by different theoretical techniques. These techniques involve the study of the different contributions which lead from a pure ionic description to the final $a b$ initio selfconsistent-field wave function, the study of the covalent effects in the metal core-level binding-energy shifts, and the analysis of uncorrelated and correlated wave functions. All the analyses are consistent with an increase of covalence in the $\mathrm{TiO}, \mathrm{Ti}_{2} \mathrm{O}_{3}, \mathrm{TiO}_{2}$ series as expected from chemical intuition.

The systematic study above reported has also permitted us to quantify the degree of ionicity along this series. The most ionic oxide has been found to be TiO but even in this case the covalent contribution to the bond is not negligible at all. Our results suggest that covalent effects are $15 \%$ or $10 \%$ larger than in the isomorphic alkalineearth oxides. Likewise, the present results point out a considerable contribution of covalence in $\mathrm{Ti}_{2} \mathrm{O}_{3}$, again larger than in corundum. As shown in a recent work, corundum exhibits a rather large ionic character; using similar techniques corundum was shown to be $\approx 90 \%$ ionic; the isomorphic $\mathrm{Ti}_{2} \mathrm{O}_{3}$ is only $78 \%$ ionic.

Overall, the ab initio cluster-model approach is well suited to predict different physical situations; from a very large ionic character in alkaline-earth oxides and corundum to an important degree of covalence in rutile. This adds further support to the idea that the high ionicity found in alkaline-earth oxides and corundum is due to a real physical effect and not to cluster-size artifacts. In fact, the degree of ionicity in rutile is rather far from the fully ionic situation. Both uncorrelated and correlated $a b$ initio wave functions indicate that rutile cannot be described as an ionic oxide. Using the terms of valencebond theory, rutile is better described as a resonance between different structures where $\mathrm{Ti}$ has an instantaneous charge of $+4,+3,+2$, and possibly +1 . While the present study cannot discriminate among these structures it is clear that the one with a net charge of +4 has only $\approx 55 \%$ contribution to the final wave function. Our conclusions are based on a finite cluster model of the bulk oxides assuming that the nature of the chemical bond is a local property. This point of view is reinforced by the close similarity between the present results for $\mathrm{TiO}_{2}$ and 
those reported from periodic Hartree-Fock calculations. $^{16}$

In conclusion, titanium oxides present a considerable amount of covalent character. This grows from $\mathrm{TiO}$ to $\mathrm{TiO}_{2}$. The use of the $a b$ initio cluster-model approach combined with different theoretical techniques has permitted us to quantify the degree of ionic character, showing that while $\mathrm{TiO}$ can be described as an ionic compound $\mathrm{TiO}_{2}$ is better viewed as a rather covalent oxide.

\section{ACKNOWLEDGMENTS}

Financial support from CICyT Project No. PB920766-C02-01 of the Spanish "Ministerio de Educación y Ciencia" is acknowledged. Part of the computer time was provided by the "Centre de Supercomputació de Catalunya (CESCA)."
${ }^{1}$ P. A. Cox, Transition Metal Oxides. An Introduction to Their Electronic Structure and Properties (Oxford University Press, New York, 1992).

${ }^{2}$ R. L. Kurtz and V. E. Henrich, Phys. Rev. B 25, 3563 (1982).

${ }^{3}$ W. H. Strelow and E. L. Cook, J. Phys. Chem. Ref. Data 2, 163 (1973).

${ }^{4}$ T. A. Sasaki and T. Soga, Physica 111B, 304 (1981).

${ }^{5}$ R. H. Tait and R. V. Kasowski, Phys. Rev. B 20, 5478 (1979).

${ }^{6}$ V. E. Henrich, G. Dresselhaus, and H. J. Zeiger, Phys. Rev. Lett. 36, 1335 (1976).

${ }^{7}$ S. Hüfner and G. K. Wertheim, Phys. Rev. B 8, 4857 (1973).

${ }^{8}$ B. W. Veal and A. P. Paulikas, Phys. Rev. B 31, 5399 (1985).

${ }^{9}$ G. Van der Laan, C. S. Mythen, and H. A. Padmore, Europhys. Lett. 11, 67 (1990).

${ }^{10}$ L. A. Grunes, R. D. Leapman, C. N. Wilker, R. Hoffman, and A. B. Kunz, Phys. Rev. B 25, 7157 (1982).

${ }^{11}$ R. Brydson, B. G. Williams, W. Engel, H. Sauer, E. Zeitler, and J. M. Thomas, Solid State Commun. 64, 609 (1987).

${ }^{12}$ J. K. Burdett, Inorg. Chem. 24, 2244 (1985).

${ }^{13}$ P. I. Sorantin and K. Schwarz, Inorg. Chem. 31, 567 (1992).

${ }^{14}$ K. M. Glassford, N. Troullier, J. L. Martins, and J. R. Chelikowsky, Solid State Commun. 76, 635 (1990).

${ }^{15}$ K. M. Glassford and J. R. Chelikowsky, Phys. Rev. B 46, 1284 (1992).

${ }^{16}$ B. Silvi, N. Fourati, R. Nada, and C. R. A. Catlow, J. Phys. Chem. Solids 52, 1005 (1991).

${ }^{17}$ A. Fahmi, C. Minot, B. Silvi, and M. Causá, Phys. Rev. B 47, 11717 (1993).

${ }^{18}$ L. B. Lin, S. D. Mo, and D. L. Lin, J. Phys. Chem. Solids 54, 907 (1993).

${ }^{19}$ R. W. G. Wyckoff, Crystal Structures (Interscience, New York, 1963), Vol. 2.

${ }^{20}$ C. Sousa, J. Casanovas, F. Illas, and J. Rubio, J. Comput. Chem. 14, 680 (1993)

${ }^{21}$ G. Pacchioni, G. Cogliandro, and P. S. Bagus, Int. J. Quantum Chem. 42, 1115 (1992).

${ }^{22}$ Gaussian Basis Sets for Molecular Calculations, edited by S. Huzinaga, Physical Science Data Vol. 16 (Elsevier, Amsterdam, 1984).

${ }^{23} \mathrm{Ph}$. Durand and J. C. Barthelat, Theor. Chim. Acta 38, 283 (1975); M. Pelissier and Ph. Durand, ibid. 55, 43 (1980).

${ }^{24}$ B. Huron, P. Rancurel, and J. P. Malrieu, J. Chem. Phys. 75,
5745 (1973).

${ }^{25}$ S. Evangelisti, J. P. Daudey, and J. P. Malrieu, Chem. Phys. Lett. 75, 91 (1983).

${ }^{26}$ F. Illas, J. Rubio, J. M. Ricart, and P. S. Bagus, J. Chem. Phys. 95, 1877 (1991).

${ }^{27}$ A. Povill, J. Rubio, and F. Illas, Theor. Chim. Acta 82, 229 (1992).

${ }^{28}$ R. Caballol and J. P. Malrieu, Chem. Phys. Lett. 188, 543 (1992).

${ }^{29}$ M. Dupuis, J. Rys, and H. F. King, HONDO-76, program 338, Quantum Chemistry Program Exchange, University of Indiana, Bloomington, IN. Pseudopotential adaptation by J. P. Daudey and M. Pelissier. General restricted open HartreeFock adaptation by R. Caballol and J. P. Daudey. CIPSI chain of programs by M. Pellisier, J. P. Daudey, J. P. Malrieu, S. Evangelisti, F. Spiegelmann, D. Maynau, J. Rubio, and F. I1las.

${ }^{30}$ P. S. Bagus, K. Hermann, and C. W. Bauschlicher, J. Chem. Phys. 81, 1966 (1984).

${ }^{31}$ P. S. Bagus, K. Hermann, and C. W. Bauschlicher, J. Chem. Phys. 80, 4378 (1984).

32P. S. Bagus and F. Illas, J. Chem. Phys. 96, 8962 (1992).

${ }^{33}$ G. Pacchioni, C. Sousa F. Illas, P. S. Bagus, and F. Parmigiani, Phys. Rev. B 48, 11573 (1993).

${ }^{34}$ C. Sousa, F. Illas, and G. Pacchioni, J. Chem. Phys. 99, 6819 (1993).

${ }^{35}$ C. Sousa, T. Minerva, G. Pacchioni, P. S. Bagus, and F. Parmigiani, J. Electron Spectrosc. Relat. Phenom. 63, 189 (1993).

${ }^{36}$ P. S. Bagus, G. Pacchioni, C. Sousa, T. Minerva, and F. Parmigiani, Chem. Phys. Lett. 196, 641 (1992).

${ }^{37}$ F. Parmigiani, G. Pacchioni, F. Illas, and P. S. Bagus, J. Electron Spectrosc Relat. Phenom. 59, 255 (1992).

${ }^{38}$ P. S. Bagus, G. Pacchioni, and F. Parmigiani, Chem. Phys. Lett. 207, 569 (1993).

${ }^{39}$ A. Clotet, J. M. Ricart, C. Sousa, and F. Illas, J. Electron Spectrosc. Relat. Phenom. (to be published).

${ }^{40}$ F. Illas, A. Lorda, J. Rubio, J. B. Torrance, and P. S. Bagus, J. Chem. Phys. 99, 389 (1993).

${ }^{41}$ A. Lorda, F. Illas, J. Rubio, and J. B. Torrance, Phys. Rev. B 47, 6207 (1992). 
(a)

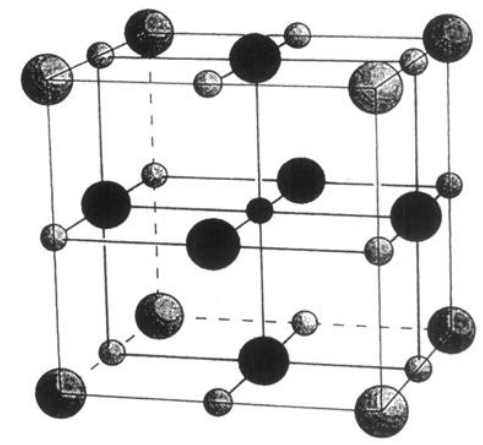

(b)

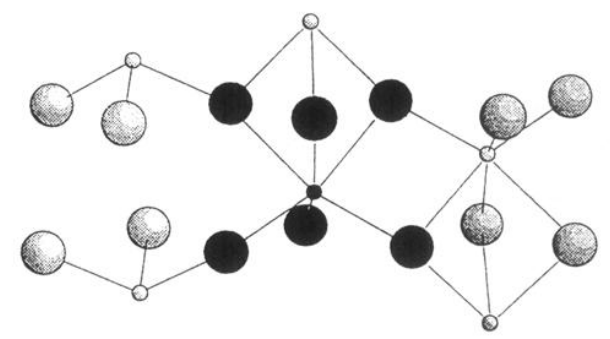

(c)

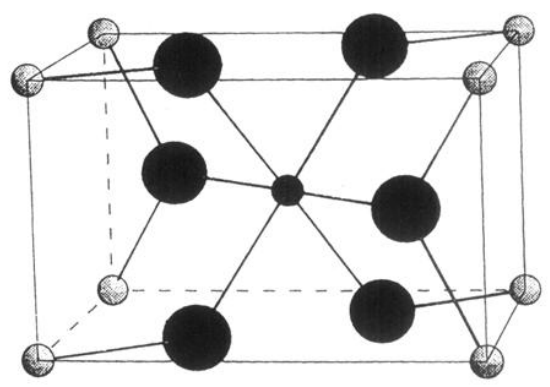

FIG. 1. Representation of the cluster models used to represent (a) $\mathrm{TiO}$, (b) $\mathrm{Ti}_{2} \mathrm{O}_{3}$, and (c) $\mathrm{TiO}_{2}$. Filled circles correspond to the ions in the cluster model and empty circles to some of the point charges used to provide an appropriate environment. Small and large circles represent the Ti cations and $\mathrm{O}^{2-}$ anions, respectively. 